\title{
Paraxial group
}

\author{
Miguel A. Bandres ${ }^{1, *}$ and Manuel Guizar-Sicairos ${ }^{2}$ \\ ${ }^{1}$ California Institute of Technology, Pasadena, California 91125, USA \\ ${ }^{2}$ The Institute of Optics, University of Rochester, Rochester, New York 14627, USA \\ *Corresponding author: bandres@caltech.edu
}

Received September 3, 2008; accepted September 27, 2008;

posted October 16, 2008 (Doc. ID 101079); published December 19, 2008

We introduce the paraxial group, the group of symmetries of the paraxial-wave equation and its action on paraxial beams. The transformations, elements of the group, are used to obtain closed-form expressions for the propagation of any paraxial beam through misaligned $A B C D$ optical systems. We prove that any paraxial beam is form-invariant under these transformations. (C) 2008 Optical Society of America

OCIS codes: $070.2580,070.2590,070.7345,260.1960$.

Finding and characterizing analytical solutions to the paraxial-wave equation (PWE) has proven to be important, not only in finding novel 3D distributions of light [1-6], but also to better understand their interaction with matter through analytical expressions for their energy, axial intensity, momentum, and angular momentum distribution. Because for practical applications the beams are typically delivered through an optical system, knowing how a beam is transformed by paraxial $A B C D$ optical systems has generated increasing interest. Even for optics with high numerical aperture, paraxial beams provide a valuable first-order approximation to understand misalignment and diffraction effects.

The PWE is equivalent to the $2 \mathrm{D}$ potential-free Schrödinger equation upon the identification of $t$ $=z m / \hbar k$. Although the group of symmetries of the later equation has been studied and characterized in the context of quantum mechanics [7] and geometrical optics [8], its application to paraxial beams is missing from wave-optics literature.

In this Letter, we introduce the group of symmetries of the PWE: the paraxial group. This group comprises the transformation of a beam by propagation through misaligned (tilted, translated, or rotated) $A B C D$ optical systems. We show that the propagation of any paraxial beam through a misaligned $A B C D$ optical system can be obtained, in closed form, in terms of the functions that describe its free-space propagation, i.e., any paraxial beam is forminvariant under these transformations.

An important difference between the Schrödinger and the paraxial group is that, in the former, the operators have real-valued parameters because they must be unitary to preserve the probability normalization. The paraxial group, on the other hand, admits complex-valued parameters that represent transformations that change the energy of the beam, e.g., apodization.

The paraxial approximation of a propagating beam is given by $U(\mathbf{r}, z)=\exp (i k z) \Psi(\mathbf{r}, z)$, where $\Psi(\mathbf{r}, z)$ satisfies the PWE

$$
\left(\nabla_{\perp}^{2}+i 2 k \frac{\partial}{\partial z}\right) \Psi(\mathbf{r}, z)=0,
$$

$\mathbf{r}=[x, y]^{T}$ is the set of transverse coordinates, $z$ is the longitudinal coordinate, $k$ is the wavenumber, and $\nabla_{\perp}^{2}$ is the transverse Laplacian.

We define the paraxial group as a Lie group of operators acting on the space of solutions of Eq. (1), i.e., mapping paraxial beams into paraxial beams. The paraxial group is composed of three subgroups. We will describe each of the subgroups and how they act on paraxial beams. We then describe how we combine the three subgroups to form the paraxial group.

The first subgroup is the group of $A B C D$ axially symmetric optical systems, which is isomorphic to the $S L(2$, C) group: the group of $2 \mathrm{D}$ complex-valued matrices with unit determinant.

If a closed-form expression for the free-space propagation of a paraxial beam is available, $\Psi(\mathbf{r}, z)$, we can readily obtain the transverse field distribution after propagation through an $A B C D$ optical system without solving Collins diffraction integral. To show this, we decompose the $A B C D$ matrix as

$$
\mathbf{M}_{A B C D}=\mathbf{M}_{I} \mathbf{M}_{P}=\left[\begin{array}{cc}
A & 0 \\
C & 1 / A
\end{array}\right]\left[\begin{array}{cc}
1 & B / A \\
0 & 1
\end{array}\right],
$$

where $\mathbf{M}_{P}$ is a free-space propagation operator and $\mathbf{M}_{I}$ is an imaging operator with magnification $m=A$. It can be shown that the propagation of a transverse field distribution through $\mathbf{M}_{I}$ is given by

$$
I_{C}\left(\mathbf{M}_{I}\right) \Psi\left(\mathbf{r}, z_{0}\right)=\frac{1}{A} \exp \left(\frac{i k C r^{2}}{2 A}\right) \Psi\left(\mathbf{r} / A, z_{0}\right),
$$

where we denote Collins diffraction integral [9] as $I_{C}\left(\mathbf{M}_{A B C D}\right)$, an operator with the $A B C D$ matrix as argument that acts on an initial transverse field distribution $\Psi\left(\mathbf{r}, z_{0}\right)$ and outputs the transverse field after propagation through the $A B C D$ optical system.

Using Eq. (3), the fact that we know the free-space propagation of the beam, and the decomposition $I_{C}\left(\mathbf{M}_{A B C D}\right)=I_{C}\left(\mathbf{M}_{I}\right) I_{C}\left(\mathbf{M}_{P}\right)$, we obtain the output transverse field after propagation through an arbitrary axially symmetric $A B C D$ system,

$$
I_{C}\left(\mathbf{M}_{A B C D}\right) \Psi(\mathbf{r}, 0)=\frac{1}{A} \exp \left(\frac{i k C r^{2}}{2 A}\right) \Psi\left(\frac{\mathbf{r}}{A}, \frac{B}{A}\right),
$$

where, without loss of generality, we assumed that $z=0$ at the input and output planes of the $A B C D$ optical system. Evaluation of the right-hand side of Eq. 
(4) requires an expression for the free-space propagation of the input beam, i.e., $\Psi(\mathbf{r}, z)$.

Although Eq. (4) appears to diverge for $A=0$ (the Fourier transform relation), the limit $A=0$ can be obtained in general, as long as the beam decays at least as $|r|^{-1 / 2}$ when $r \rightarrow \infty$.

We obtain the output beam, after propagating through an $A B C D$ optical system, by applying a freespace propagation after the $A B C D$ transformation, i.e., $P\left(\mathbf{M}_{A B C D}\right)=I_{C}\left(\mathbf{M}_{1, z, 0,1} \mathbf{M}_{A B C D}\right)$,

$$
\begin{aligned}
P\left(\mathbf{M}_{A B C D}\right) \Psi(\mathbf{r}, z)= & \frac{1}{A+C z} \exp \left[\frac{i k C r^{2}}{2(A+C z)}\right] \\
& \times \Psi\left(\frac{\mathbf{r}}{A+C z}, \frac{B+D z}{A+C z}\right),
\end{aligned}
$$

where $\mathbf{M}_{A B C D}$ does not depend on $z$ and the righthand side of Eq. (5) is a solution of the PWE. The group product is given by the product of the individual matrices $P(\mathbf{M}) P\left(\mathbf{M}^{\prime}\right)=P\left(\mathbf{M M}^{\prime}\right)$.

The second subgroup is the translation group $\mathcal{T}$. The parameters of the translation group (in general complex-valued) are a position space vector $\mathbf{v}$ $=\left[v_{x}, v_{y}\right]^{T} \in \mathrm{C}$, a momentum space vector $\mathbf{u}=\left[u_{x}, u_{y}\right]^{T}$ $\in \mathrm{C}$, and a dimensionless scalar $\rho \in \mathrm{C}$. The action of an element of the translation group $T(\mathbf{u}, \mathbf{v}, \rho) \in \mathcal{T}$ over a paraxial beam is given by

$$
\begin{aligned}
T(\mathbf{u}, \mathbf{v}, \rho) \Psi(\mathbf{r}, z)= & \exp \left[i \rho+\frac{i}{2}\left(2 \mathbf{r}-\frac{z}{k} \mathbf{u}\right) \cdot \mathbf{u}\right] \\
& \times \Psi(\mathbf{r}-z \mathbf{u} / k+\mathbf{v}, z) .
\end{aligned}
$$

Physically, $\mathbf{v}$ and $\mathbf{u}$ represent a translation of the beam in position and momentum space, respectively. Notice that a real-valued translation in momentum space is equivalent to imparting a tilt by an angle $\sin \theta=u / k$ on the paraxial beam. The imaginary part of a complex-valued translation in momentum space represents an exponential apodization of the beam, given by $\exp [-\mathbf{r} \cdot \operatorname{Im}(\mathbf{u})]$. Conversely, a complexvalued translation in position space implies an exponential apodization of the beam's Fourier spectrum. The group product is given by

$T(\mathbf{u}, \mathbf{v}, \rho) T\left(\mathbf{u}^{\prime}, \mathbf{v}^{\prime}, \rho^{\prime}\right)=T\left(\mathbf{u}+\mathbf{u}^{\prime}, \mathbf{v}+\mathbf{v}^{\prime}, \rho+\rho^{\prime}+\mathbf{u}^{\prime} \cdot \mathbf{v}\right)$.

The third subgroup is the rotation group, $S O(2)$, which rotates any paraxial beam around the optical axis. The action of $R(\theta) \in S O(2)$, is given by

$$
R(\theta) \Psi(\mathbf{r}, z)=\Psi(\Theta \mathbf{r}, z),
$$

where $\theta \in[0,2 \pi)$ and

$$
\boldsymbol{\Theta}(\theta)=\left[\begin{array}{cc}
\cos \theta & -\sin \theta \\
\sin \theta & \cos \theta
\end{array}\right] .
$$

The group product is given by $R(\theta) R\left(\theta^{\prime}\right)=R\left(\theta+\theta^{\prime}\right)$.

We construct the paraxial group, from these three subgroups, in the following way. Using the action of the groups over paraxial beams it is easy to show that the rotation group commutes with the $S L(2, \mathrm{C})$ group, i.e., $P^{-1} R^{-1} P R=1$. We can then take the direct product of these groups to make a larger group $\mathcal{W}$ $=S L(2, \mathrm{C}) \times S O(2)$. In the same way, we can see that although $\mathcal{T}$ does not commute with $\mathcal{W}$, it is invariant under conjugation with $\mathcal{W}$, i.e., $W^{-1} T W \in \mathcal{T} \forall W$ $\in \mathcal{W}, T \in \mathcal{T}$. The action of $\mathcal{W}$ over $\mathcal{T}$ is given by

$$
\begin{aligned}
R(-\theta) T(\mathbf{u}, \mathbf{v}, \rho) R(\theta) & =T(\Theta \mathbf{u}, \Theta \mathbf{v}, \rho), \\
P\left(\mathbf{M}^{-1}\right) T(\mathbf{u}, \mathbf{v}, \rho) P(\mathbf{M}) & =T\left(\mathbf{u}^{\prime}, \mathbf{v}^{\prime}, \rho^{\prime}\right),
\end{aligned}
$$

where

$$
\begin{aligned}
& \mathbf{u}^{\prime}=A \mathbf{u}+k C \mathbf{v}, \\
& \mathbf{v}^{\prime}=D \mathbf{v}+B \mathbf{u} / k, \\
& \rho^{\prime}=\rho+\left(\mathbf{u}^{\prime} \cdot \mathbf{v}^{\prime}-\mathbf{u} \cdot \mathbf{v}\right) / 2 .
\end{aligned}
$$

We can then take the semidirect product $(\rtimes)$ of $\mathcal{W}$ and $\mathcal{T}$ to obtain the paraxial group,

$$
\mathcal{G}=\mathcal{T} \rtimes[S L(2, \mathrm{C}) \times S O(2)],
$$

where the group elements $G \in \mathcal{G}$ are

$$
G(\mathbf{M}, \theta, \mathbf{u}, \mathbf{v}, \rho)=P(\mathbf{M}) R(\theta) T(\mathbf{u}, \mathbf{v}, \rho) .
$$

The action of the paraxial group over $\Psi(\mathbf{r}, z)$ is

$$
\begin{aligned}
G(g) \Psi(\mathbf{r}, z)= & \frac{1}{A+C z} \exp \left[\frac{i k C r^{2}}{2(A+C z)}\right] \\
& \times \exp \left\{i \rho+\frac{i}{2(A+C z)}\right. \\
& \times[2 \Theta \mathbf{r}-(B+D z) \mathbf{u} / k] \cdot \mathbf{u}\} \\
& \times \Psi\left(\frac{\Theta \mathbf{r}-(B+D z) \mathbf{u} / k}{A+C z}+\mathbf{v}, \frac{B+D z}{A+C z}\right),
\end{aligned}
$$

where $g=\{\mathbf{M}, \theta, \mathbf{u}, \mathbf{v}, \rho\}$ is the set of group parameters. Finally, the group product is $G(g) G\left(g^{\prime}\right)=G\left(g_{f}\right)$, where

$$
\begin{aligned}
& g_{f}=\left\{\mathbf{M M} \mathbf{M}^{\prime}, \theta+\theta^{\prime}, \mathbf{u}_{f}, \mathbf{v}_{f}, \rho_{f}\right\}, \\
& \mathbf{u}_{f}=\mathbf{u}^{\prime}+\Theta^{\prime}(\theta)\left(A^{\prime} \mathbf{u}+k C^{\prime} \mathbf{v}\right), \\
& \mathbf{v}_{f}=\mathbf{v}^{\prime}+\Theta^{\prime}(\theta)\left(D^{\prime} \mathbf{v}+B^{\prime} \mathbf{u} / k\right), \\
& \rho_{f}=\rho+\rho^{\prime}+\frac{1}{2}\left[\left(\mathbf{u}_{f}+\mathbf{u}^{\prime}\right) \cdot\left(\mathbf{v}_{f}-\mathbf{v}^{\prime}\right)-\mathbf{u} \cdot \mathbf{v}\right] .
\end{aligned}
$$

Equation (15) is the first important result of this Letter; it shows how the paraxial group maps any paraxial beam to a new paraxial beam.

For example, with our formalism the results presented in [1-4] for the propagation of circular, Cartesian, Airy, and Helmholtz-Gauss (HzG) beams, through an $A B C D$ optical system can be found in a 
straightforward manner without the need for evaluating Collins diffraction integral. This is even more important for beams whose functions are mathematically more complex such as the accelerating parabolic beams [3] or elliptical beams [6].

The propagation of paraxial beams through a misaligned optical system can be treated by the generalized diffraction integral given in [9]. However, an equivalent result can be obtained without solving any integrals using the paraxial group. A misaligned optical system is characterized by a displacement vector, $\boldsymbol{\varepsilon}=\left[\varepsilon_{x}, \varepsilon_{y}\right]^{T}$, the inclined angles, $\varepsilon^{\prime}=\left[\varepsilon_{x}^{\prime}, \varepsilon_{y}^{\prime}\right]^{T}$, the $A B C D$ transfer matrix elements of the aligned optical system, and the air-equivalent axial distance between the input and output planes of the aligned $A B C D$ system $\left(L=\Sigma_{i} L_{i} / n_{i}\right)$ [9].

The beam after propagation through a misaligned optical system can be obtained by

$$
\begin{aligned}
& G\left[\mathbf{I}, 0, k \boldsymbol{\varepsilon}^{\prime},-\boldsymbol{\varepsilon}-L \boldsymbol{\varepsilon}^{\prime},-k \boldsymbol{\varepsilon}^{\prime} \cdot\left(\boldsymbol{\varepsilon}+L \boldsymbol{\varepsilon}^{\prime}\right)\right] \\
& \quad \times G\left(\mathbf{M}_{A B C D}, 0, \mathbf{0}, \mathbf{0}, 0\right) G\left(\mathbf{I}, 0,-k \boldsymbol{\varepsilon}^{\prime}, \boldsymbol{\varepsilon}, 0\right) \Psi(\mathbf{r}, z),
\end{aligned}
$$

where the first transformation takes the beam to the misaligned reference frame, the second transformation propagates the beam through the aligned $A B C D$ system, and the third transformation brings the beam back to the original reference frame.

Using the paraxial group product rule in Eq. (16), Eq. (17) can be reduced to a single transformation

$$
\begin{gathered}
G\left\{\mathbf{M}_{A B C D}, 0, k(\mathbf{e}+A \mathbf{g}) / B, \mathbf{g},-k \boldsymbol{\varepsilon}^{\prime} \cdot\left(\boldsymbol{\varepsilon}+L \boldsymbol{\varepsilon}^{\prime}\right) / 2\right. \\
\left.+\left[k(\mathbf{e}+A \mathbf{g}) / B-k \boldsymbol{\varepsilon}^{\prime}\right] \cdot(\mathbf{g}-\boldsymbol{\varepsilon}) / 2\right\} \Psi(\mathbf{r}, z),
\end{gathered}
$$

where the parameters $\mathbf{e}$ and $\mathbf{g}$ take the form

$$
\begin{aligned}
& \mathbf{e}=\left(\alpha \boldsymbol{\varepsilon}+\beta \boldsymbol{\varepsilon}^{\prime}\right), \\
& \mathbf{g}=(B \gamma-D \alpha) \boldsymbol{\varepsilon}+(B \delta-D \beta) \boldsymbol{\varepsilon}^{\prime},
\end{aligned}
$$

and the misaligned matrix elements are given by

$$
\left[\begin{array}{ll}
\alpha & \beta \\
\gamma & \delta
\end{array}\right]=\left[\begin{array}{cc}
1-A & L-B \\
-C & 1-D
\end{array}\right] .
$$

The transformation given by Eq. (18) is the second important result of this Letter. Together with Eq. (15) they show that the propagation of any paraxial beam through a misaligned $A B C D$ optical system can be obtained in closed form, in terms of the functions that describe its free-space propagation.

Consider, for example, the misaligned two lens system shown in Fig. 1(a), where both lenses are biconvex (same curvature on front and back surfaces), have an index $n=1.5$, and a thickness of $1 \mathrm{~cm}$. The beam after the first lens, $\Psi_{1}(\mathbf{r}, z)$, is obtained by applying the transformation given in Eq. (18), with $\varepsilon$ $=[0, \Delta y]^{T}$ and $\boldsymbol{\varepsilon}^{\prime}=\mathbf{0}$, over $\Psi_{0}\left(\mathbf{r}, z+t_{0}\right)$, i.e., $\Psi_{1}(\mathbf{r}, z)$ $=G \Psi_{0}\left(\mathbf{r}, z+t_{0}\right)$. The beam after the second lens is readily obtained by evaluating $\Psi_{2}(\mathbf{r}, z)=G \Psi_{1}(\mathbf{r}, z$ $+t_{1}$ ) using the transformation given in Eq. (18) with $\boldsymbol{\varepsilon}=\mathbf{0}$ and $\boldsymbol{\varepsilon}^{\prime}=\left[\Delta \phi_{x}, 0\right]^{T}$. For both cases, $\mathbf{M}$ is the
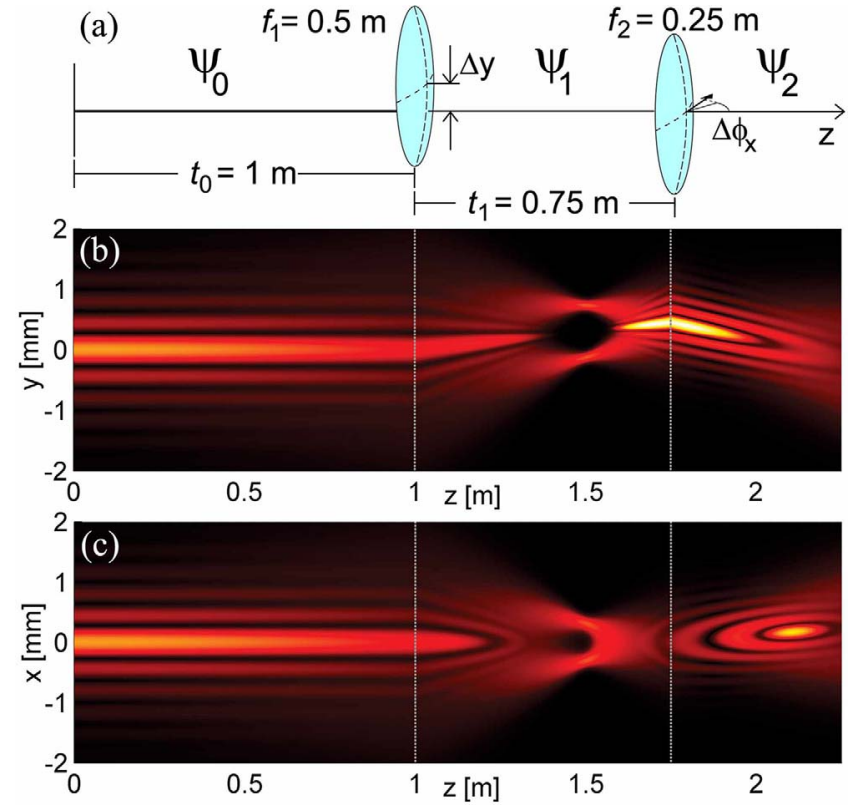

Fig. 1. (Color online) (a) Misaligned optical system. (b) and (c) show the propagation of a $\mathrm{HzG}$ beam through the optical system for $\Delta y=3 \mathrm{~mm}$ and $\Delta \phi_{x}=2^{\circ}$. Dashed lines indicate the position of the lenses.

$A B C D$ matrix for the corresponding thick lens [9]. The propagation of a $\mathrm{HzG}$ beam through this optical system is shown in Figs. 1(b) and 1(c). The input beam, $\Psi_{0}(\mathbf{r}, z)$, is a zeroth-order Bessel-Gauss beam [1] with $\lambda=632.8 \mathrm{~nm}, k_{t}=\pi k / 3600$, and $w_{0}=1.2 \mathrm{~mm}$.

Additionally, our formulation can be used to obtain new solutions for the PWE through the use of complex-valued parameters. For example, ideal nondiffracting beams or ideal accelerating beams cannot be created experimentally because they carry infinite energy but can be made square-integrable by a Gaussian $[\operatorname{Im}(C)>0]$ or exponential $[\operatorname{Im}(\mathbf{u}) \neq 0]$ apodization, respectively [1,3].

M. A. Bandres acknowledges support from the Secretaría de Educación Publica de México.

\section{References}

1. M. Guizar-Sicairos and J. C. Gutiérrez-Vega, Opt. Lett. 31, 2912 (2006).

2. M. A. Bandres and J. C. Gutiérrez-Vega, Opt. Lett. 32, 3459 (2007).

3. M. A. Bandres and J. C. Gutiérrez-Vega, Opt. Express 15, 16719 (2007).

4. M. A. Bandres and J. C. Gutiérrez-Vega, Opt. Lett. 33, 177 (2008).

5. M. A. Bandres, Opt. Lett. 33, 1678 (2008).

6. M. A. Bandres and J. C. Gutiérrez-Vega, "Elliptical beams" (submitted to Opt. Express).

7. W. Miller, Symmetry and Separation of Variables (Cambridge U. Press, 1984).

8. K. B. Wolf, Geometric Optics on Phase Space (Springer, 2004).

9. W. Shaomin and L. Ronchi, in Progress in Optics, Vol. XXV, E. Wolf, ed. (Elsevier, 1988), pp. 281-348. 